\title{
Real gas state equations comparative analysis for low- temperature calculations
}

\author{
Mikhail Sokolov ${ }^{1, *}$, Nikolay Sadovsky ${ }^{1}$, Anatoly Zuev ${ }^{1}$, Lyubov Gileva ${ }^{1}$, and Minh Hai Nguyen ${ }^{2}$ \\ ${ }^{1}$ Peter the Great St. Petersburg Polytechnic University, Polytechnicheskaya 29, St. Petersburg, 195251, Russian Federation \\ ${ }^{2}$ Petrovietnam Exploration Production Corporation, Ho Chi Minh City, Viet Nam
}

\begin{abstract}
In this paper, various real gas state equations are considered and their comparative analysis is carried out. The following state equations are studied in the work: Benedict-Webb-Rubin modification equation, Ridlich-Kwong Real Gas equation, Peng-Robinson Real Gas equation, and the modified RidlichKwong real gas state equations proposed by Barsuk S.D. We have made a direct comparison of these calculation methods with most accurate identification. In addition, the paper analyzes the equations features, with applicability limits definition of each state equations. For the chosen one, as the most universal and exact equation, the calculations were made for the liquid phase and the real gas two-phase state. Based on the data obtained, polynomials were developed for various parameters depending on the gas temperature, which can later be used to build various mathematical models. Our conclusions show main advantages of selected equation for real gases and the reasons for choosing it for modeling low-temperature heat and mass transfer processes.
\end{abstract}

\section{Introduction}

The basis for most modern design, calculation or analysis of gas equipment is the calculation of the thermodynamics of the working environment. By gas equipment is meant directly gas, as well as oil and gas, chemical and other equipment working with gas or a two-phase liquid-gas medium, including: compressors, turbines, heat exchangers and more. Under the working environment is meant real gas in a gas, liquid or adjacent phase. Thermodynamic calculation refers directly to the calculation of the thermodynamic parameters of a real gas, including compressibility, density, temperature, pressure, etc.

The issue of calculating the thermodynamics of the working environment is relevant only for the calculation of real gases. In thermodynamics, a real gas is understood to mean any gas whose properties cannot be determined by the Mendeleev-Klaiperon equation with sufficient accuracy. The main features of real gas are: high pressure, low temperature, specific gas composition. To solve the problems of designing, calculating or analyzing equipment with a similar working environment, it is necessary to apply the equations of state of real gas.

At the moment, there is a huge number of different equations of state of the real gas for various practical problems. These methods can vary the temperature and pressure ranges at which they can be applied with high accuracy, direct accuracy, as well as the list of gases that this method takes into account.
In this work, we will carry out: the implementation of equations of state of a real gas on a computer, an analysis of the accuracy indicators of the equations of state, a comparative analysis of the accuracy and efficiency of the equations of state, the identification of various features of the equations of state. The comparison will take into account such parameters as the accuracy of the equation within the limits of applicability of the equation, the accuracy of the equation beyond the limits of applicability, the ranges of applicability of the equation for temperature and pressure, the composition of the gases taken into account in the equation, the features of calculating secondary parameters. Secondary parameters are understood as the thermodynamic properties of gases, to obtain which it is necessary to apply additional calculations that are not related to the equation of state of a real gas.

The following equations of state of a real gas will be considered in the work: a modified Benedict-WebbRubin gas equation of state, proposed by the All-Russian Research Institute of Natural Gas (BWR) [1,2], the Ridlich-Kwong equation of state (RK) [3], the PengRobinson equation of state (PR) [3], a modification of the Ridlich-Kwong equation proposed by Barsuk S.D. (RKB) [4]. Also, some equations of state excluded from comparison will be described in the paper and the rationale for this exception from comparison will be given [5].

The purpose of the study: to identify the most effective equations of state of real gas for solving problems of designing, calculating or analyzing gas equipment, identifying the main parameters of various

\footnotetext{
* Corresponding author: smi1994spb@gmail.com
} 
equations of state, such as accuracy, range of applicability, the gases taken into account and various calculation features, using the above equations of state for calculating gas equipment, for example, a lowtemperature methane compressor.

\section{Methods}

\subsection{Modification of the Benedict-Webb-Rubin equation of state of real gas proposed by the All-Russian Research Institute of Natural Gas (BWR)}

This equation of state was derived from extrapolation of experimental data on the thermophysical parameters of the pure components of a mixture of real gas, which means that for the correct calculation using this empirical-analytical computational method of thermodynamic calculation of real gas, it is necessary to know the individual coefficients for each such component included in the desired mixture, and their interaction with each other or the amount, in terms of physical interaction within the mixture, according to this method is taken into account closer nym equations taken from either approximate law states or from the combination of rules for gas mixtures [6].

Eight equation constants are usually calculated from volumetric data for pure components. At the moment, there are several sets of constants of the Benedict-WebbRubin equation, as well as modifications of this equation, for various problems and calculations [5,7].

The modified BWequation in its basic form is as follows:

$$
\begin{aligned}
& Z^{3}-Z^{2}-Z \cdot\left(\frac{a_{1}}{\tau}-\frac{a_{2}}{\tau^{2}}-\frac{a_{3}}{\tau^{4}}\right) \cdot \pi+ \\
& +\left(\frac{a_{4}}{\tau^{2}}-\frac{a_{5}}{\tau^{3}}+\frac{a_{6}}{\tau^{5}}\right) \cdot \pi^{2}=0
\end{aligned}
$$

The following equation follows from this:

$$
\begin{aligned}
& Z=1+\left(\frac{a_{1}}{\tau}-\frac{a_{2}}{\tau^{2}}-\frac{a_{3}}{\tau^{4}}\right) \cdot \frac{\pi}{Z}+ \\
& +\left(\frac{a_{4}}{\tau^{2}}-\frac{a_{5}}{\tau^{3}}+\frac{a_{6}}{\tau^{5}}\right) \cdot \frac{\pi^{2}}{Z^{2}}
\end{aligned}
$$

where $Z$ is the compressibility coefficient, $\pi$ is the reduced pressure, $\tau$ is the reduced temperature, $a_{1} \ldots a_{6}$ are the coefficients of the BWR equation [1].

To calculate the reduced thermodynamic parameters, it is necessary to use the following functions $[1,8,9,10]$ :

$$
\pi=\frac{P}{P_{c r}},
$$

$$
\tau=\frac{T}{T_{c r}},
$$

where $P$ is the real gas pressure, $P_{c r}$ is the critical pressure of the real gas for the pure element and the mean critical (pseudocritical) pressure for the gas mixture, $T$ is the temperature of the real gas, $T_{c r}$ is the critical temperature of the real gas for the pure element and the mean critical (pseudocritical) temperature for the gas.

The average critical parameters are calculated based on individual indicators of the critical characteristics and the molar fraction of the substance in the mixture $[1,8,9,10]$ :

$$
\begin{aligned}
& P_{c r}=\sum_{i=1}^{n} P_{c r i} \cdot y_{i}, \\
& T_{c r}=\sum_{i=1}^{n} T_{c r i} \cdot y_{i},
\end{aligned}
$$

where $P_{c r i}$ is the individual critical pressure of the mixture component, $y_{i}$ is the molar fraction of the component in the mixture.

To solve the gas state equation directly, it is enough to use the compressibility coefficient obtained above in the Mendeleev-Klaiperon equation:

$$
\frac{P}{\rho}=z \cdot R \cdot T \text {. }
$$

where $\rho$ is the density of the real gas, $R$ is the individual gas constant of the real gas.

In this form, the BWR equation has a strictly limited range of applicability: $260 \mathrm{~K}<\mathrm{T}<400 \mathrm{~K}$; $\mathrm{P}<15 \mathrm{MPa}$. Testing and verification of this equation was carried out on four basic gas compositions with a percentage of methane from $100 \%$ to $85 \%$. The table below shows the compositions of the tested real gases, for which the experimental data were extrapolated [1].

In addition, the dependences of heat capacity and enthalpy using the above BWR constants were derived for this equation, which expands the possibilities of applying this equation [1]. Thus, we can say that this equation of state of real gas is mainly suitable for solving the problems of designing, calculating or analyzing gas equipment at moderate temperatures and pressures and at a high methane content in the working medium, for example, for calculating compressors and separators operating with natural gas.

Of course, this equation of state does not apply to low temperature calculations. However, based on the high degree of validation and elaboration of this equation at the Department of Campressor Engineering, it was decided to include this equation in the comparison. Comparison of the equations of state of the real gas in the range of applicability of the BWR equation will help 
Table 1. The composition of the experimental gases.

\begin{tabular}{|c|c|c|c|c|c|}
\hline Name of gas & $\begin{array}{c}\text { Chemical } \\
\text { formula }\end{array}$ & $\begin{array}{c}\text { The mole fraction in } \\
\text { pure methane, \% }\end{array}$ & $\begin{array}{c}\text { Molar fraction according } \\
\text { to GOST 23194, \% }\end{array}$ & $\begin{array}{c}\text { Molar fraction } \\
\text { in gas №.1, \% }\end{array}$ & $\begin{array}{c}\text { Molar fraction } \\
\text { in gas №. 2, \% }\end{array}$ \\
\hline Methane & $\mathrm{CH}_{4}$ & 100 & 98.63 & 93.30 & 85.95 \\
\hline Ethane & $\mathrm{C}_{2} \mathrm{H}_{6}$ & - & 0.12 & 4.00 & 5.85 \\
\hline Propane & $\mathrm{C}_{3} \mathrm{H}_{8}$ & - & 0.02 & 0.60 & 2.07 \\
\hline n-butane & $\mathrm{n}_{-} \mathrm{C}_{4} \mathrm{H}_{10}$ & - & 0.10 & 0.40 & 0.74 \\
\hline n-Pentane and the higher & $\mathrm{n}_{-}-\mathrm{C}_{5} \mathrm{H}_{12}+$ & - & - & 0.30 & 0.36 \\
\hline Carbon dioxide & $\mathrm{CO}_{2}$ & - & 1.01 & 0.10 & 2.19 \\
\hline Nitrogen & $\mathrm{N}_{2}$ & - & 0.12 & 1.30 & 2.84 \\
\hline
\end{tabular}

to validate and analyze all other equations in the same range. And studying the BWR equation in lowtemperature calculations will help to collect the necessary data for analysis and possible improvement of this equation for specific technological problems.

\subsection{Ridlich-Kwong Real Gas Equation (RK)}

This equation, published in 1949, had the following form:

$$
p=\frac{R \cdot T}{v-b}-\frac{a}{T^{0,5} \cdot v \cdot(v+b)},
$$

where a, b are the coefficients obtained from the conditions at the critical point.

The coefficients themselves are the following functions:

$$
a, b=f\left(R, T_{c}, p_{c}\right)
$$

And since all three values on which these coefficients depend (critical pressure, critical temperature, individual gas constant) are constants with a constant gas composition, it will be sufficient to calculate these coefficients for each substance over the entire volume of temperatures and pressures, which greatly simplifies multivariate calculations.

On average, this method and its modifications, despite a fairly accurate description of the thermodynamic processes of real gas themselves, have a rather high indicator of relative calculation error. So, for a multicomponent gas mixture at temperatures in the range from $73 \mathrm{~K}$ to $413 \mathrm{~K}$ and at pressures from 0.15 $\mathrm{MPa}$ to $14 \mathrm{MPa}$, provided that the pressure of the mixture does not exceed more than 0.9 of the critical pressure of this mixture, the calculation results reach deviations of 30-50\% from experimental values. [3]

This method is well applicable for high temperatures (above 0.6 from critical) and for two-component mixtures, where its accuracy is close to the previously considered BWR method.

\subsection{Peng-Robinson Real Gas Equation (PR)}

The basis of this equation of state of a real gas was the widespread, at the time of the creation of the PR method (1975), van der Waals equation [3].

This equation has the form:

$$
p=\frac{R \cdot T}{v-b}-\frac{a}{v \cdot(v+b)+b \cdot(v-b)}
$$

At the same time, a very important innovation in this equation was the revision of the method for calculating the coefficient a, which, as a result, became dependent not only on the three above constants, but also on the current temperature of the medium and the coefficient of acentricity of the substance.

The introduction of this feature, as well as the additional term $b \cdot(v-b)$, had an extremely positive effect on the growth of calculation accuracy when using the PR method.

It is extremely important to note the fact that both of the above equations are able to calculate the phase transitions of a substance and multiphase mixtures, and are also able to take into account the humidity of the gas.

\subsection{The modified Ridlich-Kwong equation of state of real gas proposed by Barsuk S.D. (RKB)}

The basis of this modification is the equation of state of the real Redlich-Kwong gas in the following form:

$$
p=\frac{R \cdot T}{v-b}-\frac{R \cdot T \cdot b \cdot d}{v \cdot(v+b)}
$$

where b, $d$ are the coefficients obtained from the conditions at the critical point. [4]

The coefficient of the equation of state of a real gas $b$ is calculated as follows:

$$
b=\sum_{i=1}^{n} b_{i} \cdot x_{i}
$$

where $\mathrm{x}$ is the molar for the substance in the mixture. [4] 
In this case, the individual value of the coefficient $b$ for each individual gas is calculated according to the equation:

$$
b_{i}=0,0867 \cdot R \cdot T_{\kappa p i} / P_{\kappa p i} .
$$

In turn, the coefficient of the equation of state of a real gas $\mathrm{d}$ is calculated, calculated as follows:

$$
d=\sum_{i=1}^{n} d_{i} \cdot x_{i},
$$

In this case, the individual value of the coefficient $d$ for each individual gas is calculated according to the equation:

$$
d_{i}=A_{i}+B_{i} \cdot\left(T_{\kappa p i} / T-1\right)+C_{i} \cdot\left(T_{\kappa p i} / T-1\right)^{2},
$$

Unlike the original Redlich-Kwong equation, in this modification, in addition to the parameters T_cr and P_cr characterizing each individual component of the mixture, there is also a coefficient $\mathrm{d}$, which depends on the reduced temperature and three individual numerical coefficients for the substance: A, B and C. [4,11,12]

This method is mainly used in the calculation of twophase mixtures (gas-liquid) for low and ultra-low temperatures. It is worth noting that the RKB is able to take into account phase transitions and gas humidity.

\subsection{Other equations of state}

\subsubsection{Lee-Kesler equation}

Separately, among the many modifications of the Benedict-Webb-Rubin equation of state, it is worth noting the generalization modification, and the most famous and widely used among these modifications is the modified Benedict-Webb-Rubin real gas state equation in the dimensionless form proposed by Lee and Kesler, or the equation Lee-Keslra [2].

This equation of state of a real gas was derived using various physical and mathematical methods, including using three-parameter Pitzer correlation [7]. In this method, the compressibility factor of a real gas $\mathrm{z}$ is associated with some reference substance, often noctane, and the empirical-analytical computational method of thermodynamic calculation of real gas is based on calculating the deviation from this norm due to some difference between the real calculated gas and the standard.

In general terms, the Lee-Kesler equation is as follows [2]:

$$
Z=Z^{(0)}+\omega \cdot Z^{(1)},
$$

where $Z^{(0)}$ is the compressibility coefficient of the "main" substance;

$$
Z^{(1)}=\frac{\left(Z^{(\ni)}-Z^{(0)}\right)}{\omega^{(\ni)}} .
$$

here $Z^{(3)}$ is the compressibility coefficient of the "reference" substance; $\omega$ is the coefficient of acentricity, $\omega^{\left({ }^{()}\right.}$is the coefficient of acentricity of the reference substance [2].

The equation of state of a real Li-Kesler gas for practical use requires significantly less information about the composition of this gas than the equation of state of Benedict-Webb-Rubin. To implement the desired calculation, you need a set of data, states from the data for the two substances studied, the reference and the current, as well as the coefficient of acentricity for the substance in question.

Basically, the Li-Kesler equation of state is used to calculate the gas and gas-liquid mixture in the following ranges of thermodynamic characteristics of real gas: 0.3 $<\operatorname{Tr}<4 ; 0<\operatorname{Pr}<10$. Thus, these limits can be calculated for a set of main gases.

For example, for pure methane, this boundary corresponds to the following parameters: $\mathrm{p}=0-46 \mathrm{MPa}$; $\mathrm{T}=57-762 \mathrm{~K}$.

For pure n-pentane: $\mathrm{p}=0$ - $34 \mathrm{MPa}$; $\mathrm{T}=141-1879 \mathrm{~K}$.

For pure isooctane: $\mathrm{p}=0-25 \mathrm{MPa} ; \mathrm{T}=171-2274 \mathrm{~K}$.

\subsubsection{Zagoruchenko Method}

Method V.A. Zagoruchenko can be described as a method of compiling equations of state for multicomponent, most often two or three-component, mixtures of real gases, implemented to determine the properties of natural and associated petroleum gas, as well as some other real gases [13].

Thus, the empirical-analytical computational method for the thermodynamic calculation of real gas, based on the Zagoruchenko method, has a number of conceptual differences from the two previous methods, namely: V.A. Zagoruchenko, as the name implies, serves precisely as a method for compiling the equations that were given in previous chapters, naturally not in form but in purpose.

The state equations of a real gas mixture are compiled in the empirical-analytical computational method of thermodynamic calculation of real gas, based on the Zagoruchenko method, according to previously found component equations and the interaction functions of the components.

It is known that the equation of state of a gas mixture can have the same form as the equation of components [13]. Based on the equation of state of the gas mixture recorded in virial form [13]:

$$
p \cdot V=R \cdot T+B \cdot \rho+C \cdot \rho^{2}+D \cdot \rho^{3}+\ldots
$$

and theoretical relations connecting the virial coefficients of the equation of state with the corresponding virial coefficients of the equations of the components and the virial coefficients of interaction, a 
general expression is obtained for determining the compressibility of the gas mixture through the equations of the components and the interaction function [13].

In particular, for a three-component mixture:

$$
\begin{aligned}
& p \cdot V=x_{1} \cdot(p \cdot V)_{1}+x_{2} \cdot(p \cdot V)_{2}+ \\
& +x_{3} \cdot(p \cdot V)_{3}+x_{1} \cdot x_{2} \cdot F_{12}(\rho, T)+ \\
& +x_{1} \cdot x_{3} \cdot F_{13}(\rho, T)+x_{2} \cdot x_{3} \cdot F_{23}(\rho, T)+ \\
& +x_{1} \cdot x_{2}^{2} \cdot F_{122}(\rho, T)+\ldots
\end{aligned}
$$

Here $(p \cdot V)_{i}$ is the compressibility of pure substances at the same temperatures and densities, $\mathrm{F}$ are functions that depend on density and temperature and take into account the pairwise interactions of particles of dissimilar gases [13].

The factors of the second and higher degrees can be neglected due to the high order of smallness and the extremely low influence on the final calculation result.

A check performed on a large number of mixtures confirms that in the overwhelming majority of cases, sufficient accuracy in the description of the thermal properties of mixtures in a wide range of densities is ensured when only pair interactions of component particles are taken into account.

This method allows you to calculate the following set of gases: methane, ethane, propane, n-butane, isobutane, 1-butene (butylene), 2-methylpropane (isobut.), Npentane, 2-methylbutane (isopent.), 2.2 demethylpropane, 1,2-butadiene (divinyl), 2-methyl-1,3butadiene, ethene (ethylene), propene (propylene), ethine (acetylene), carbon dioxide, oxygen, carbon monoxide, nitrogen, hydrogen [13].

At the same time, the range of permissible pressures and temperatures is up to $70 \mathrm{MPa}$ and $150-700 \mathrm{~K}$, respectively.

\section{Results and Discussion}

Next, a comparative calculation of the BWR, PR and RK methods will be given, as well as a comparison of the results of PR and RK calculations with the data obtained in the calculation in the RefProp software module used in industrial calculations.

Table 2 presents comparisons of the 4th limit points calculated for BWR when calculating pure methane.

As can be seen from the comparison, cold spots at high pressure by the RK method have an extremely high deviation from the other two methods.

Table 3 presents the results of calculating PR and RK with the data obtained during the calculation in the RefProp software module.

As can be seen from the table, the deviation of RK relative to RefProp is significantly higher than the deviation of PR and RCB relative to RefProp. It should be understood that the data obtained in RefProp also have some error relative to experimental indicators, however, the deviation of RK exceeds the deviation of other methods by almost three times.

It is also obvious that, on average, the deviation indices of PR and RCB are close, however, the PR method, when solving our particular problem, has several advantages over the RCB method:

The RKB method is dependent on the individual coefficients of substances, which greatly limits the possibilities of its modernization and expansion to nonstandard gas compositions, as a result of which, the PR method has a wider range of substances to which it can be applied.

Below are the data obtained using the PR equation and their processing for further use in another calculation environment.

The main condition for a transition of this kind is the compilation of polynomials for the main parameters of the gas, depending on temperature at constant pressure or depending on temperature and pressure.

Since any real gas has three characteristic sections

Table 2. Comparative calculation of BWR, RK, PR and RKB.

\begin{tabular}{|c|c|c|c|c|c|}
\hline Point № & Parameter & BWR & RK & PR & RKB \\
\hline \multirow{4}{*}{ Point 1 } & $\mathrm{P}, \mathrm{MPa}$ & 1 & 1.0367 & 0.99908 & 0.99624 \\
\cline { 2 - 6 } & $\mathrm{T}, \mathrm{K}$ & 270 & 270 & 270 & 270 \\
\cline { 2 - 6 } & $\rho, \mathrm{kg} / \mathrm{m}^{3}$ & 7.3282 & 7.3282 & 7.3282 & 7.3282 \\
\hline \multirow{3}{*}{ Point 2 } & $\mathrm{P}, \mathrm{MPa}$ & 10 & 15.2543 & 10.6008 & 9.6685 \\
\cline { 2 - 6 } & $\mathrm{T}, \mathrm{K}$ & 270 & 270 & 270 & 270 \\
\cline { 2 - 6 } & $\rho, \mathrm{kg} / \mathrm{m}^{3}$ & 92.6158 & 92.6158 & 92.6158 & 92.6158 \\
\hline \multirow{3}{*}{ Point 3 } & $\mathrm{P}, \mathrm{MPa}$ & 1 & 1.01882 & 0.99677 & 0.99985 \\
\cline { 2 - 6 } & $\mathrm{T}, \mathrm{K}$ & 350 & 350 & 350 & 350 \\
\cline { 2 - 6 } & $\rho, \mathrm{kg} / \mathrm{m}^{3}$ & 5.5666 & 5.5666 & 5.5666 & 5.5666 \\
\hline \multirow{3}{*}{ Point 4 } & $\mathrm{P}, \mathrm{MPa}$ & 10 & 11.9729 & 9.86014 & 10.004 \\
\cline { 2 - 6 } & $\mathrm{T}, \mathrm{K}$ & 350 & 350 & 350 & 350 \\
\cline { 2 - 6 } & $\rho, \mathrm{kg} / \mathrm{m}^{3}$ & 59.4598 & 59.4598 & 59.4598 & 59.4598 \\
\hline
\end{tabular}


Table 3. Comparative calculation of RK, PR, RKB, RefProp.

\begin{tabular}{|c|c|c|c|c|c|}
\hline Point № & Parameter & BWR & RK & PR & RKB \\
\hline \multirow{3}{*}{ Point 1} & $\mathrm{P}, \mathrm{MPa}$ & 1.8901 & 2.4056 & 2.00179 & 1.84847 \\
\hline & $\mathrm{T}, \mathrm{K}$ & 180 & 180 & 180 & 180 \\
\hline & $\rho, \mathrm{kg} / \mathrm{m}^{3}$ & 25 & 25 & 25 & 25 \\
\hline \multirow{3}{*}{ Point 2} & $\mathrm{P}, \mathrm{MPa}$ & 2.0429 & 2.54257 & 2.13767 & 1.95117 \\
\hline & $\mathrm{T}, \mathrm{K}$ & 190 & 190 & 190 & 190 \\
\hline & $\rho, \mathrm{kg} / \mathrm{m}^{3}$ & 25 & 25 & 25 & 25 \\
\hline \multirow{3}{*}{ Point 3} & $\mathrm{P}, \mathrm{MPa}$ & 2.1938 & 2.67945 & 2.27356 & 2.05386 \\
\hline & $\mathrm{T}, \mathrm{K}$ & 200 & 200 & 200 & 200 \\
\hline & $\rho, \mathrm{kg} / \mathrm{m}^{3}$ & 25 & 25 & 25 & 25 \\
\hline \multirow{3}{*}{ Point 4} & $\mathrm{P}, \mathrm{MPa}$ & 2.3433 & 2.81626 & 2.40945 & 2.15655 \\
\hline & $\mathrm{T}, \mathrm{K}$ & 210 & 210 & 210 & 210 \\
\hline & $\rho, \mathrm{kg} / \mathrm{m}^{3}$ & 25 & 25 & 25 & 25 \\
\hline \multirow{3}{*}{ Point 5} & $\mathrm{P}, \mathrm{MPa}$ & 2.4917 & 2.953 & 2.54533 & 2.25924 \\
\hline & $\mathrm{T}, \mathrm{K}$ & 220 & 220 & 220 & 220 \\
\hline & $\rho, \mathrm{kg} / \mathrm{m}^{3}$ & 25 & 25 & 25 & 25 \\
\hline \multirow{3}{*}{ Point 6} & $\mathrm{P}, \mathrm{MPa}$ & 2.6391 & 3.08969 & 2.68122 & 2.36194 \\
\hline & $\mathrm{T}, \mathrm{K}$ & 230 & 230 & 230 & 230 \\
\hline & $\rho, \mathrm{kg} / \mathrm{m}^{3}$ & 25 & 25 & 25 & 25 \\
\hline \multirow{3}{*}{ Point 7} & $\mathrm{P}, \mathrm{MPa}$ & 2.7857 & 3.22633 & 2.81711 & 2.46463 \\
\hline & $\mathrm{T}, \mathrm{K}$ & 240 & 240 & 240 & 240 \\
\hline & $\rho, \mathrm{kg} / \mathrm{m}^{3}$ & 25 & 25 & 25 & 25 \\
\hline \multirow{3}{*}{ Point 8} & $\mathrm{P}, \mathrm{MPa}$ & 3.077 & 3.49948 & 3.08888 & 2.67002 \\
\hline & $\mathrm{T}, \mathrm{K}$ & 260 & 260 & 260 & 260 \\
\hline & $\rho, \mathrm{kg} / \mathrm{m}^{3}$ & 25 & 25 & 25 & 25 \\
\hline \multirow{3}{*}{ Point 9} & $\mathrm{P}, \mathrm{MPa}$ & 3.222 & 3.63599 & 3.22477 & 2.77271 \\
\hline & $\mathrm{T}, \mathrm{K}$ & 270 & 270 & 270 & 270 \\
\hline & $\rho, \mathrm{kg} / \mathrm{m}^{3}$ & 25 & 25 & 25 & 25 \\
\hline \multirow{3}{*}{ Point 10} & $\mathrm{P}, \mathrm{MPa}$ & 3.3665 & 3.77247 & 3.36065 & 2.87540 \\
\hline & $\mathrm{T}, \mathrm{K}$ & 280 & 280 & 280 & 280 \\
\hline & $\rho, \mathrm{kg} / \mathrm{m}^{3}$ & 25 & 25 & 25 & 25 \\
\hline \multirow{3}{*}{ Point 11} & $\mathrm{P}, \mathrm{MPa}$ & 3.5106 & 3.90892 & 3.49654 & 2.97810 \\
\hline & $\mathrm{T}, \mathrm{K}$ & 290 & 290 & 290 & 290 \\
\hline & $\rho, \mathrm{kg} / \mathrm{m}^{3}$ & 25 & 25 & 25 & 25 \\
\hline
\end{tabular}

(liquid, gas-liquid, gas), three different polynomials will be provided for each substance. Table 4 provides a description of the characteristics of the gas, which contains $26.47 \%$ octane and $73.53 \%$ n-pentane $[14,15,16,17,18]$.

Figures 1-5 show graphs of the dependences of density on substance temperature.

Based on the data, the polynomial of this section has a linear form. We plot for the mixing site.
This section can be conditionally divided into two, the first of which corresponds to a sharp decline, and the third of which is practically invariant to temperature changes, so we get the following three dependencies.

Thus, we obtain the following relationships presented in table 5 . 
Table 4. Description of gas characteristics.

\begin{tabular}{|c|c|c|c|c|c|}
\hline Temperature, K & Pressure, MPa & Density, $\mathbf{~ k g} / \mathbf{m}^{\mathbf{3}}$ & Enthalpy, $\mathbf{~ J J / k g}$ & Entropy, $\mathbf{~ k J / k g}$ *K & Phase state \\
\hline 250 & 0.15 & 652.41 & -79.414 & -0.296 & Liquid \\
\hline 260 & 0.15 & 643.03 & -58.836 & -0.2153 & Liquid \\
\hline 270 & 0.15 & 633.5 & -37.8 & -0.13591 & Liquid \\
\hline 280 & 0.15 & 623.79 & -16.289 & -0.05769 & Liquid \\
\hline 290 & 0.15 & 613.85 & 5.7141 & 0.019517 & Liquid \\
\hline 295 & 0.15 & 608.79 & 16.906 & 0.057779 & Liquid \\
\hline 299 & 0.15 & 604.69 & 25.952 & 0.088239 & Liquid \\
\hline 299.4 & 0.15 & 604.28 & 26.861 & 0.091278 & Liquid \\
\hline 299.4 & 0.15 & 604.28 & 26.869 & 0.091302 & Wet steam \\
\hline 300 & 0.15 & 42.398 & 58.936 & 0.1983 & Wet steam \\
\hline 305 & 0.15 & 8.6631 & 193.85 & 0.64502 & Wet steam \\
\hline 310 & 0.15 & 6.7628 & 243.09 & 0.80536 & Wet steam \\
\hline 320 & 0.15 & 5.8733 & 285 & 0.9386 & Wet steam \\
\hline 330 & 0.15 & 5.4118 & 318.64 & 1.0421 & Wet steam \\
\hline
\end{tabular}

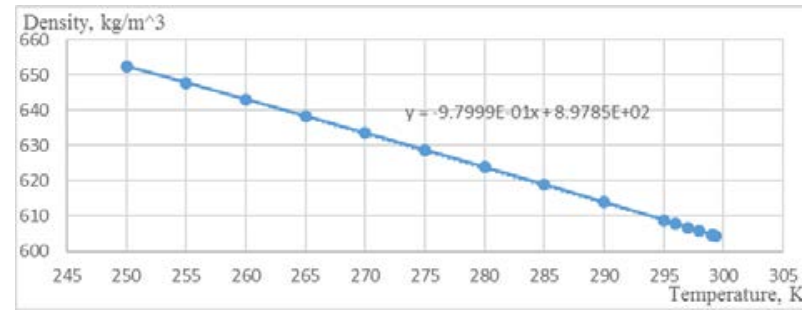

Fig. 1. Graph of the liquid phase of the test substance.

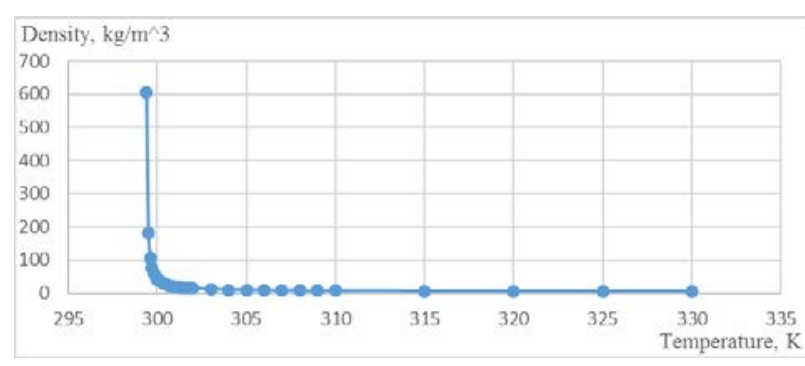

Fig. 2. Graph of the Gas-liquid phase of the test substance.

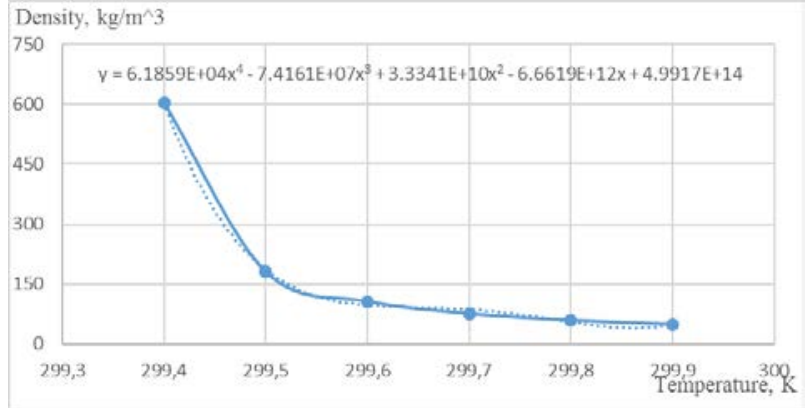

Fig. 3. Schedule of the first section.

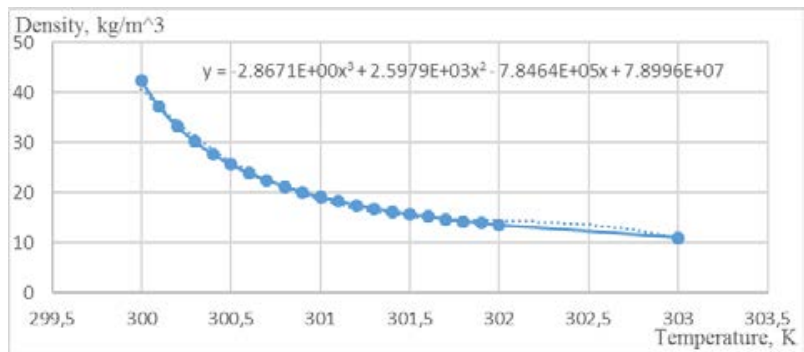

Fig. 4. Graph of the second section.

Table 5. Equations of characteristic sections of the state of the gas.

\begin{tabular}{|c|c|c|}
\hline $\begin{array}{c}\text { Minimum } \\
\text { temperature, } \mathbf{K}\end{array}$ & $\begin{array}{c}\text { Maximum } \\
\text { temperature, } \mathbf{K}\end{array}$ & The equation \\
\hline 250 & 299.4 & $\mathrm{y}=-9.7999 \mathrm{E}-01 \mathrm{x}+8.9785 \mathrm{E}+02$ \\
\hline 299.4 & 299.9 & $\mathrm{y}=6.1859 \mathrm{E}+04 \mathrm{x}^{4}-7.4161 \mathrm{E}+07 \mathrm{x}^{3}+3.3341 \mathrm{E}+10 \mathrm{x}^{2}-6.6619 \mathrm{E}+12 \mathrm{x}+4.9917 \mathrm{E}+14$ \\
\hline 300 & 303 & $\mathrm{y}=-2.8671 \mathrm{E}+00 \mathrm{x}^{3}+2.5979 \mathrm{E}+03 \mathrm{x}^{2}-7.8464 \mathrm{E}+05 \mathrm{x}+7.8996 \mathrm{E}+07$ \\
\hline 303 & 330 & $\mathrm{y}=-7.8194 \mathrm{E}-04 \mathrm{x}^{3}+7.5229 \mathrm{E}-01 \mathrm{x}^{2}-2.4125 \mathrm{E}+02 \mathrm{x}+2.5792 \mathrm{E}+04$ \\
\hline
\end{tabular}




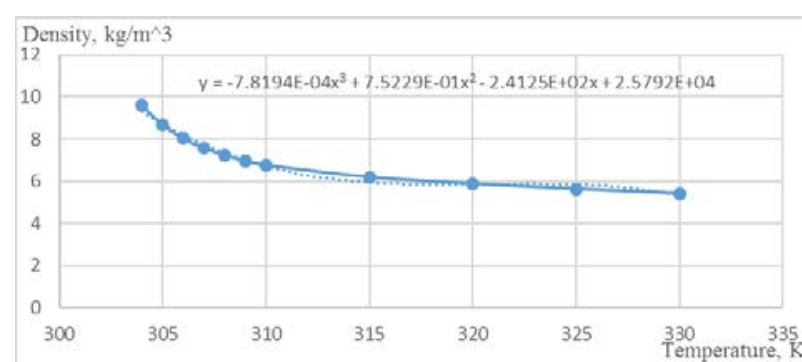

Fig. 5. Graph of the third section.

\section{Conclusions}

Considering all the above methods, it was decided to use the RK method for further calculations and the implementation of the analytical calculation model of a real gas for the following reasons [19,20,21]:

1) The RK method is able to take into account phase transitions in the calculation of real gas, which is one of the main requirements for future calculation;

Often, modeling low-temperature processes is closely related to the calculation of the vapor-liquid and liquid phases of various substances. The ability to account for such special operating conditions is critical to many processes.

2) The RK method has an extremely high range of applicability in terms of temperature, pressure and gas composition;

This feature allows the PR method to be the most versatile in comparison with the rest.

3) Moreover, this equation has high accuracy over the entire range of applicability;

4) The equation also has a gigantic amount of various modifications that will make it possible to correct the method in the future when strong deviations occur.

In fact, none of the proposed methods has all of the above advantages with respect to the PR method.

\section{References}

1. Schurovsky V.A., Sinitsyn Yu.N., Korneev V.I., Cheremin A.V., Stepanov G.S.: PR 51-31323949-43-99. Guidelines for conducting thermal engineering and gasdynamic calculations during testing of gas turbine gas pumping units, 26. VNIIGAS, M. (1999)

2.Yun V.K.: Fundamentals of improving design methods and unification of centrifugal compressors for various purposes: the dissertation ... Doctors of technical sciences: 05.04.06. Yun Vladimir Klimentevich; [Place of defense: GOUVPO" St. Petersburg State Polytechnic University"] St. Petersburg (2012)

3. Brusilovsky A.I.: Phase transformations in the development of oil and gas fields. M.: “Grail”, 575 (2002)

4. Barsuk S.D.: Calculation of the thermodynamic properties of natural gas. News of the USSR Academy of Sciences, 124-131 (1981)

5. Sadovskiy, N., Strizhak, L., Simonov, A., Sokolov, M.: Some problem of centrifugal compressors upgrading. MATEC Web of Conferences, 245, article 09004. DOI: 10.1051/matecconf/201824509004 (2018)

6. Reed R., Prausnits J., Sherwood T.: Properties of gases and liquids. Reference manual. Per. from English 3rd ed. L.: Chemistry (1982)

7.Sychev V.V.: et al. Thermodynamic properties of methane M., Publishing house of standards, 348 (1979)

8. GOST 30319.2-96. Natural gas. Methods for calculating physical properties. Determination of compressibility coefficient 44 (2015)

9.ISO/TC 193 SC1 63. Natural gas calculation of compression factor. Part 3: Calculation using measured physical properties

10.ISO/TC 193 SC1 62. Natural gas calculation of compression factor. Part 2: Calculation using a molar composition analysis

11. Barsuk S.D., Surkov Yu.V., Benjaminovich O.A., Schelkunova L.D.: Guidelines for calculating the thermodynamic properties of natural gas. M.: ed. VNIIGAZ,16 (1975)

12. Sirotin S.A., Mamaev A.V., Kopsha D.P.: The choice of the equation of state for modeling the processes of liquefaction of natural gas. SCIENCE \& TECHNOLOGY IN THE GAS INDUSTRY 4 from. 5964 (2018)

13. Zagoruchenko V.A.: The study of thermodynamic properties and charting the state of natural gases and their main components in relation to the tasks of compressor engineering: dis. Doct. tech. sciences. Odessa, 267 (1964)

14. Danilishin A.M., Aksenov A.A., Kozhukhov Y.V., Simonov A.M.: Investigation of Inaccuracy of the Three-Dimensional Viscous Turbulent Flow Modeling in the Flow Path of the Two-Element Centrifugal Compressor Stage. Territorija "NEFTEGAS" [Oil and Gas Territory](3), 44-55 (2019)

15. Ivanov E.A., Zharkovsky A.A., Borshchev I.O., Svoboda D.G.: Technique for axial pump characteristics predicting in CFD package Openfoam. AIP Conference Proceedings v.2141,\#030063. (2019). DOI: 10.1063/1.5122113

16. Zhurkin N., Donskoj A., Zharkovskij A.: Numeric modeling and estimating the performance characteristics of a pneumatic driven high pressure pump. MATEC Web of Conferences, 245, \# 09014. DOI: 10.1051/matecconf/201824509014 (2018)

17.A.A. Zharkovskii A.Y. Pospelov, Use of 3D Methods for Flow Analysis, Prediction of Characteristics, and Optimization of the Shape of Settings of Hydraulic Turbines. Power Technology and Engineering, 49(1), 27-32 (2015)

18. Pospelov A.Y., Zharkovskii A.A.: Effect of the Parameters of a Computational Model on the Prediction of Hydraulic Turbine Characteristics. Power Technology and Engineering, 49(3), 159-164 (2015)

19. Juan Sebastian Lopez-Echeverrya, Simon Reif-Achermana, Eduard Araujo-Lopez. Peng-Robinson equation of state: 40 years through cubics. Fluid Phase Equilibria (2017)

20. Motonao Imai, Huanquan Pan, Michael Connolly, Hamdi Tchelepi, Masanori Kurihara.: Reduced variables method for four-phase equilibrium 
calculations of hydrocarbon-water-CO2 mixtures at a low temperature. Fluid Phase Equilibria (2019)

21. Uskov S.I., Potemkin D.I., Shigarov A.B., Snytnikov P.V., Kirillov V.A., Sobyanin V.A.: Lowtemperature steam conversion of flare gases for various applications. Chemical Engineering Journal V. 3682019 533-540 (2019) 